Bio-grafía Escritos sobre La Biologia y su Enseñanza.

Edición Extra-Ordinaria. ISSN 2027-1034 P.p $780-804$

Memorias del VII Encuentro Nacional de Experiencias en la Enseñanza de la Biología y la Educación Ambiental y II Congreso Nacional de Investigación en la Enseñanza de La Biología

\title{
EVOLUCIÓN: CONCEPCIONES E INCIDENCIAS DEL QUEHACER DEL MAESTRO Y SUS ESTRATEGÍAS DIDÁCTICAS
}

\section{EVOLUTION: CONCEPTIONS AND INCIDENCES OF TEACHER'S TASK AND HIS TEACHING STRATEGIES}

\section{Oscar David Ríos Rodríguez ${ }^{1}$}

\section{Resumen}

La investigación descrita a continuación, tiene como objeto la identificación de concepciones alternativas de los estudiantes del IED Instituto Nacional de Promoción Social de Villeta/ Cundinamarca alrededor de la Evolución Biológica. Éstas se identifican a través del análisis de contenido, y se indaga si las concepciones pueden ser transformadas a través de las estrategias didácticas. Las principales concepciones sobre Evolución manifiestan que es un proceso de transformación de los organismos en el tiempo, así como un progreso paulatino que tienen los seres vivos hacia la perfección. El desarrollo de la propuesta didáctica incide en la transformación en las concepciones de los estudiantes hacia concepciones explicativas respecto a Evolución biológica.

Palabras Clave: Evolución, concepciones, estrategia didáctica

\section{Abstract}

The research described below, aims to identify students' alternative conceptions from IED Instituto Nacional de Promocion Social in Villeta / Cundinamarca about Biological Evolution. These are identified through content analysis, and ask whether the ideas can be transformed through teaching strategies. The main conceptions state that evolution is a process of transformation of organisms over

\footnotetext{
${ }^{1}$ Universidad Pedagógica Nacional, Departamento de Biología (Noveno semestre), Línea de investigación Biodiversidad y Conservación de los Sistemas Acuáticos de la Región Andina (SARA). Artículo de investigación producto de Práctica pedagógica. Correo Electrónico : Od2r@hotmail.com ,Dbi.orios@pedagogica.edu.co
} 
Bio-grafía Escritos sobre La Biologia y su Enseñanza.

Edición Extra-Ordinaria. ISSN 2027-1034 P.p 780 - 804

Memorias del VII Encuentro Nacional de Experiencias en la Enseñanza de la Biología y la Educación Ambiental y II Congreso Nacional de Investigación en la Enseñanza de la Biología

time and gradually progress with living beings towards perfection. The development of didactic transformation affects the conceptions of students towards explanatory conceptions about biological evolution.

Key words: Evolution, Conceptions, Teaching strategy Introducción

Los estudios sobre las concepciones alternativas han estado presentes en la investigación educativa, sobre varias áreas del cocimiento científico, incluyendo las ciencias naturales y su marco conceptual. Abarcando varios niveles educativos. Las investigaciones en el campo de la enseñanza y el aprendizaje de las ciencias experimentales se han realizado durante al menos las últimas dos décadas, principalmente en países de habla inglesa.(Vienott,1979;Driveretal,1989;Pozo, 1996;Campanario yOtero, 2000 en Fernández y Sanjosé, 2007).

En Biología, principalmente en Evolución, estos estudios se extienden a diferentes niveles educativos, formales, informales y fuera del contexto educativo. Resaltando que las concepciones más recurrentes son de carácter Teleológico. La evolución es concebida como una vía hacia el perfeccionamiento, que es acentuada en algunos libros de texto de la enseñanza de la Biología. Por otra parte la Evolución se concentra en el campo de la discordia entre el conocimiento científico y las creencias religiosas. (Fernández,J. \&Sanjosé, V., 2007)

En este sentido, el estudio sobre las concepciones alternativas se problematiza desde dos aspectos fundamentales. El primero, se refiere a la enseñanza de la Teoría Evolutiva como una teoría acabada, reductible a las definiciones del marco conceptual que la conforman. De otra parte, laTeoríaEvolutiva,propiamentedicha, se mencionan algunos de sus aspectos importantes en los estándares básicos en ciencias naturales, aún resultan insuficientes para la comprensión del proceso 
Bio-grafia Escritos sobre la Biologia y su Enseñanza.

Edición Extra-Ordinaria. ISSN 2027-1034 P.p p 780 - 804

Memorias del VII Encuentro Nacional de Experiencias en la Enseñanza de la Biología y la Educación Ambiental y II Congreso Nacional de Investigación en la Enseñanza de la Biología

evolutivo en su conjunto. A través de la caracterización de las concepciones alternativas de los estudiantes, se propone una estrategia didáctica sobre Evolución a partir del marco conceptual de Ecología Evolutiva, como alternativa de enseñanza en el contexto escolar.

\section{Materiales y métodos}

La propuesta didáctica, descrita en el documento, se diseñó y se desarrolló en dos fases principales. La primera, Fase de indagación, que tuvo como objetivo principal diagnosticar las concepciones alrededor del concepto Evolución biológica que manifiestan los estudiantes de grado Noveno del INPS. La segunda fase o fase de desarrollo conceptual, se refiere al diseño de la estrategia didáctica, el conjunto declases, actividades y evaluaciónenlacualsedesarrollala propuestadeenseñanza deEvolucióna partirdelfundamentoconceptualdela Ecología Evolutiva.

(En la tabla 1 se describen las fases metodológicas de la propuesta didáctica)

\begin{tabular}{|c|c|c|c|}
\hline $\begin{array}{c}\text { FASE } \\
1\end{array}$ & $\begin{array}{c}\text { ¿Qué es } \\
\text { Evolución? }\end{array}$ & $\begin{array}{l}\text { - } \text { Taller } \\
\text { - Ensay }\end{array}$ & $\begin{array}{l}\text { previas } \\
\text { nera Entrega }\end{array}$ \\
\hline \multirow[b]{2}{*}{$\begin{array}{c}\text { FASE } \\
2\end{array}$} & \multirow[b]{2}{*}{$\begin{array}{l}\text { Ecología } \\
\text { Evolutiva }\end{array}$} & $\begin{array}{c}\text { Selección } \\
\text { Natural }\end{array}$ & $\begin{array}{l}\text { - Selección Natural como } \\
\text { mecanismo de evolución. } \\
\text { - Selección y Adaptación. }\end{array}$ \\
\hline & & FITNESS & $\begin{array}{l}\text { - Reproducción sexual y asexual } \\
\text { - Estrategias r y K } \\
\text { - Selección sexual } \\
\text { - Cortejo } \\
\text { - Estructuras para reproducción } \\
\text { - Sexo salvaje }\end{array}$ \\
\hline
\end{tabular}


Bio-grafía Escritos sobre La Biologia y su Enseñanza.

Edición Extra-Ordinaria. ISSN 2027-1034 P.p p 780 - 804

Memorias del VII Encuentro Nacional de Experiencias en la Enseñanza de la Biología y la Educación Ambiental y II Congreso Nacional de Investigación en la Enseñanza de La Biología

\begin{tabular}{|l|l|l|l|}
\hline \multirow{2}{*}{} & & & \\
\cline { 3 - 4 } & Adaptación & $\begin{array}{l}\text { Especies especialistas y } \\
\text { especies generalistas } \\
\text { Especiación. }\end{array}$ \\
\hline
\end{tabular}

Tabla 1: Fases metodológica y ejes temáticos

Los ensayos, y demás documentos realizados por los estudiantes son sometidos a análisis de contenido, de acuerdo a esto, Bardin (1986) plantea el análisis de contenido como elconjuntodeinstrumentosmetodológicos, aplicados aloqueelautordenominacomo"discursos"extremadamentediversificados, se trata de una hermenéutica controlada basada en la deducción: "la inferencia". El instrumento metodológico empleado para el análisis de contenido es una matriz de categorización de las concepciones, a partir de las líneas de tendencia fuerza que manifiestan los estudiantes en sus escritos, esto es, la frecuencia y sentido del uso de un concepto en torno a la explicación y definición de lo que Evolución significa (Ver anexo 1 ). Cada una de las categorías está relacionada con lo que los estudiantes manifiestan

en

sus documentosapartirdelascualessehaceelanálisisdecadaunadelascategoríascontrast ándolas conelmarcoteóricoempleadoparalarealizacióndelaestrategiapedagógica,loquepermi teinferirsi las concepciones delosestudiantesestánalejadas, oporelcontrario,muycercanasalcontenido teórico.

Resultados y Análisis

"Antes de Ecología Evolutiva" 
Memorias del VII Encuentro Nacional de Experiencias en la Enseñanza de la Biología y la Educación Ambiental y II Congreso Nacional de Investigación en la Enseñanza de la Biología

Las categorías que se obtuvieron del análisis de contenido de los ensayos (1 ${ }^{\mathrm{a}}$ entrega) se construyeron a partir de la frecuencia con la que un concepto aparecía en torno al significado de Evolución manifestado por los estudiantes. Se resaltan once concepciones diferentes.

Es necesario señalar que los estudiantes manifiestan concepciones principalmente descriptivas, esto es, los estudiantes definen evolución desde su contexto, haciendo uso del lenguaje cotidiano para expresar sus ideas, sin remitirse al uso de conceptos propios de la Teoría Evolutiva. Las principales concepciones manifestadas por los estudiantes se describen en la Tabla 2.

\begin{tabular}{|c|c|}
\hline Concepcióndescriptiva & Descripcióndelaconcepción \\
\hline Transformación & $\begin{array}{l}\text { Laevoluciónimplica cambios enlos organismos } \\
\text { a través del tiempo }\end{array}$ \\
\hline Aparicióndenuevasespecies & $\begin{array}{l}\text { El hecho evolutivo es la vía por la cual } \\
\text { aparecen nuevas especies. }\end{array}$ \\
\hline Mejoramiento & $\begin{array}{l}\text { Laevoluciónconllevaalaperfecciónpaulatina } \\
\text { delos organismos }\end{array}$ \\
\hline $\begin{array}{c}\text { Cambiodecélulasaorganismosc } \\
\text { omplejos }\end{array}$ & $\begin{array}{l}\text { Laevolucióneslavíaatravésdelacuallos } \\
\text { organismoshan dejadode } \\
\text { cépulussimplesaorganjsmoscomplejoscon } \\
\text { tejidos y sistemas deórganos. }\end{array}$ \\
\hline $\begin{array}{l}\text { Cambios durante } \\
\text { lavidadeunorganismo }\end{array}$ & $\begin{array}{l}\text { La transformación quepueda tener } \\
\text { unorganișmodurantesuvida, } \\
\text { ensucrecimientoydesarrollo,particularmenteenlo } \\
\text { sorganismos quepresentanmetamorfosis. }\end{array}$ \\
\hline Cambios físicos, mentales. & $\begin{array}{l}\text { manifestacióndecomportamientosy } \\
\text { La enelser } \\
\text { conductasespecialmente } \\
\text { humanoconformesudesarrollo físicoysicológico }\end{array}$ \\
\hline Adaptación & $\begin{array}{l}\text { Laadaptaciónesresultadodela } \\
\text { evolución,peronoseexplicaa } \\
\text { cualmecanismo.Seenfocaprincipalmenésdetalo traveague } \\
\text { losorganismosporsuestructuraestanadaptadosa } \\
\text { ambientesen particular. }\end{array}$ \\
\hline Evolución: Humanos & $\begin{array}{l}\text { Laevoluciónesexclusivadelossereshumanos. Se } \\
\text { manifiestael sentido antropicodelaevolución. }\end{array}$ \\
\hline
\end{tabular}


Bio-grafia Escritos sobre la Biologia y su Enseñanza.

Edición Extra-Ordinaria. ISSN 2027-1034 P.p 780 - 804

Memorias del VII Encuentro Nacional de Experiencias en la Enseñanza de la Biología y la Educación Ambiental y II Congreso Nacional de Investigación en la Enseñanza de La Biología

\begin{tabular}{|c|l|}
\hline Avance tecnológico & $\begin{array}{l}\text { La evolución se presenta conforme la } \\
\text { tecnología y las herramientas cambian por } \\
\text { lainvención humana. }\end{array}$ \\
\hline Pasoporetapas & $\begin{array}{l}\text { Laevoluciónesunaseriedeetapasporlascualesatr } \\
\text { aviesaun organismodurantesudesarrollo. }\end{array}$ \\
\hline Necesidad & $\begin{array}{c}\text { Losorganismosevolucionanporeldeseointrínseco } \\
\text { decambiar, demejorar. }\end{array}$ \\
\hline
\end{tabular}

Tabla2:Descripcióngeneraldelasconcepcionesutilizadasporlosestudiantesale xplicarla evolución.

La evolución da a conocer los cambios físicos de los seres vivos, ya que a lo largo del tiempo van teniendo distintas transformaciones según su especie.

(...) Por consiguiente puedo deducir que la evolución es el mecanismo por el cual se obtiene los diversos cambios en cada ser vivo por medio de transformaciones que van ocurriendo a lo largo del tiempo" (E008) (E014)(Cita textual deEnsayo)

\begin{abstract}
Laevoluciónesloquenosotrosdejamoscomounaherenciaoalgoasíyeslo quehace que nosparezcamosa nuestrafamiliaporla genética, quelo vemos en elADN (...) Alo largodeltiemponosotros vamos dejando unparecidoen nuestrafamilia perosiempre vansurgiendograndescambios (E035) (Cita textual deEnsayo)
\end{abstract}

Apartirdelosresultadosobtenidosesposibleidentificarquelosestudiantesconcibenlaev olución comounconjunto detransformaciones quetienenlugarenunperíododetiempo,es portanto lacausa principaldelorigen deladiversidadbiológica, enlaquelacapacidaddelosorganismosdedejar descendencia y la herencia genética se relacionan, y que la causa de estos cambios en las 
Bio-grafía Escritos sobre La Biologia y su Enseñanza.

Edición Extra-Ordinaria. ISSN 2027-1034 P.p p 780 - 804

Memorias del VII Encuentro Nacional de Experiencias en la Enseñanza de la Biología y la Educación Ambiental y II Congreso Nacional de Investigación en la Enseñanza de la Biología

característicasdelosorganismosradicaenlaintención,eldeseointrínsecodelosorganis mospor alcanzarungradodeperfeccióncada vezmayor.

En relación a lo anterior, Fernández y Sanjosé (2007), señalan que los estudiantes aceptan la Evolución como fenómeno, eludiendo explicaciones fijistas y admiten que los seres vivos en el pasado eran diferentes de los actuales. Sin embargo las concepciones alternativas surgen cuando pretenden explicar este fenómeno.

Fernández y Sanjosé (2007), también se refieren a la persistencia de ideas lamarckianas de evolución, a causa del esfuerzo, hacia el progreso y por necesidad también la herencia de caracteres adquiridos como la causa principal de los cambios entre las especies.

Segúnmanifiestanlosestudiantes,laadaptacióncomoresultadodelaevoluciónrespond ea

lasnecesidadesdelosorganismosporhabitarydesarrollarsusfuncionesestructuralese nun

ambientedeterminado.La

adaptaciónesresultadodelprocesoevolutivo,sinembargo,los

estudiantes,nohacenreferenciaalos eventosyprocesosqueconducenaloscambios evolutivos,sinoque éstos correspondenaunanecesidaddelosorganismos por evolucionar

\section{"Después de Ecología Evolutiva"}

Con el desarrollo del componente temático propuesto a través de la estrategia didáctica, se realizó la segunda entrega de los ensayos por parte de los estudiantes, que se sometieron a análisis de contenido. La categorización se realizó en torno a dos preguntas centrales que se abordaban en los ensayos; ¿Qué es Evolución? Y ¿Cómo evolucionan los organismos?, de acuerdo a estas preguntas orientadoras se busca establecer entonces, las definiciones que los 
Memorias del VII Encuentro Nacional de Experiencias en la Enseñanza de la Biologia y la Educación Ambiental y II Congreso Nacional de Investigación en la Enseñanza de la Biología

estudiantes construyeron alrededor del concepto Evolución. En la Tabla 3 se presentan algunas de las definiciones sobre Evolución que los estudiantes construyeron luego del desarrollo temático.

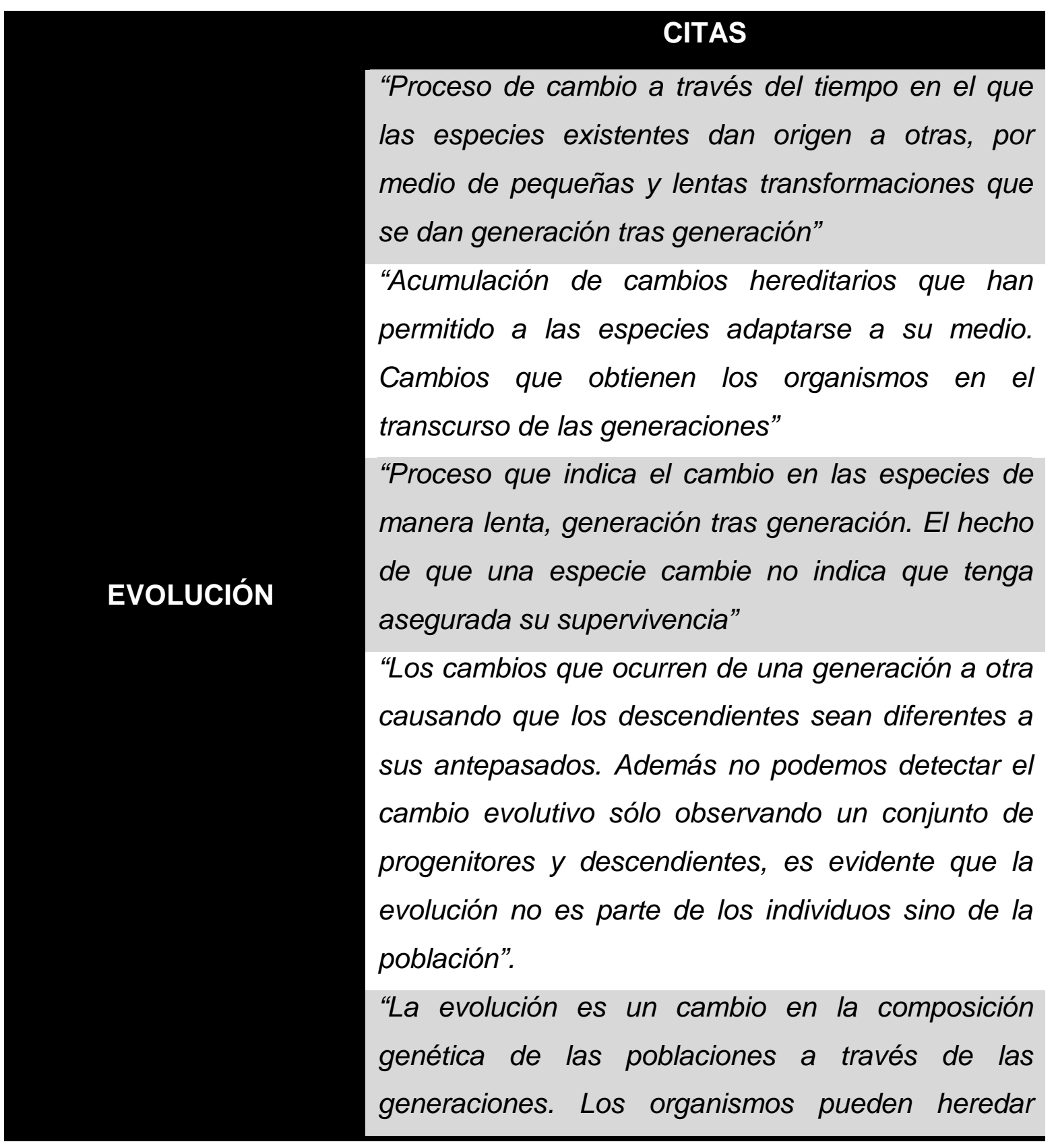


Bio-grafía Escritos sobre la Biología y su Enseñanza.

Edición Extra-Ordinaria. ISSN 2027-1034 P.p 780 - 804

Memorias del VII Encuentro Nacional de Experiencias en la Enseñanza de la Biología y la Educación Ambiental y II Congreso Nacional de Investigación en la Enseñanza de La Biología

características a sus hijos a causa de los genes que
tienen sus progenitores".

Tabla 3. Definiciones de Evolución hechas por los estudiantes

De acuerdo a lo expresado por los estudiantes a través de sus ensayos, las concepciones sobre Evolución se acercan y se ajustan al contenido conceptual en comparación a lo que manifestaban al comienzo del proceso, así mismo el tipo de concepciones se ha transformado de concepciones descriptivas, es decir las características de la evolución, hacia concepciones explicativas de lo que Evolución significa, esto es, los estudiantes acuden al conjunto de conceptos que fueron desarrollados en el abordaje conceptual para dar cuenta del cambio Evolutivo.

\section{Conclusiones}

Bartov (1978, 1981) (Citado por Gándara, M. et al., 2002) sugiere que las ideas espontáneas surgen en torno a la explicación de connotación finalista e intencional, que son posible detectarlas debido a que los estudiantes identifican propósitos con causas, en este sentido, los cambios evolutivos responden a la consecución de un fin. Estas ideas resultan resistentes incluso en estudiantes que han estudiado biología evolutiva desde temprana edad.La enseñanza tradicional no facilita la comprensión de los conceptos evolutivos, y que aún después de estos procesos en los estudiantes aún permanecen las concepciones alternativas alrededor de estos conceptos, según afirman Skoug y Biolica (2002), resultados que coinciden con otros estudios realizados alrededor de la enseñanza de la Evolución.

En este sentido, y de acuerdo a los resultados obtenidos, es posible afirmar que 
Bio-grafia Escritos sobre la Biologia y su Enseñanza.

Edición Extra-Ordinaria. ISSN 2027-1034 P.p 780 - 804

Memorias del VII Encuentro Nacional de Experiencias en la Enseñanza de la Biología y la Educación Ambiental y II Congreso Nacional de Investigación en la Enseñanza de la Biología

las concepciones alternativas son susceptibles de transformación, cuando la enseñanza del marco conceptual de la Teoría Evolutiva se plantea a partir de una estrategia didáctica que modifique la enseñanza tradicional de este campo de las ciencias. En este sentido, el maestro a través de la reflexión y la indagación sobre qué enseñar, como puntos de partida para abordar la teoría evolutiva se constituye en la materia prima del diseño de sus estrategias didácticas. Las implicaciones de las estrategias didácticas están determinadas por el nivel no sólo de planeación y diseño, sino de reflexión e indagación, sobre el cual se erigen sus estrategias, esto es, el alcance de las estrategias didácticas del maestro serán el resultado de la actividad inherente que cómo investigador debe desarrollar.

El desarrollo de las estrategias pedagógicas y la enseñanza de la biología inciden en la transformación de las concepciones de los estudiantes cuando el desarrollo de la actividad del maestro involucra a los estudiantes en el proceso de enseñanza, es decir, cuando una estrategia didáctica es diseñada a partir de las concepciones alternativas de los estudiantes, el aprendizaje de los conceptos dependerá, además de la actividad del maestro, de los intereses particulares de los estudiantes por acercarse al objeto de estudio.

La estrategia didáctica presentada en este trabajo, presenta el marco conceptual de Ecología Evolutiva como una alternativa en el diseño no solo de una estrategia, sino que concibe la posibilidad de evaluar el eje curricular de la enseñanza de la Biología en la educación escolar a través de un diseño curricular a partir de la Evolución, en tanto ésta se conciba como el eje unificador de la Biología misma.

\section{Bibliografía}

- BARDIN, L. (1986): El análisis de contenido. Madrid, Akal 
Bio-grafia Escritos sobre la Biologia y su Enseñanza.

Edición Extra-Ordinaria. ISSN 2027-1034 P.p p 780 - 804

Memorias del VII Encuentro Nacional de Experiencias en la Enseñanza de la Biología y la Educación Ambiental y II Congreso Nacional de Investigación en la Enseñanza de la Biología

- Bernedo, V., Tizón, M. C. (2011). Concepciones alternativas sobre Evolución en $1^{\circ}$ de ESO. IES Xoan Montes (Lugo)

- FERNÁNDEZ, J., SANJOSÉ, V. (2007). Permanencia de ideas alternativas sobre Evolución de las Especies en la población culta no especializada. Didáctica de las Ciencias experimentales y sociales. № 21. 2007. ISSN 02144379: 129- 149.

- Gándara, M., Gil, M.J. y Sanmartín, N. (2002). Del modelo científico de "adaptación biológica" al modelo de "adaptación biológica" en los libros de texto de Enseñanza Secundaria Obligatoria. Enseñanza de las Ciencias , 20 (2), 303-314.

- SKOUG, G., BIOLICA, K. (2002). The emphasis given to evolution in State Science Standards. A lever for change in evolution education? Science Education 4(86), 445-450.

ANEXOS

Anexo 1. Matriz de Categorías de Análisis de contenido.

\begin{tabular}{|c|c|c|c|}
\hline Categoría & Citas & Análisis & $\begin{array}{c}\text { Estudiantes } \\
\text { totales/ } \\
\text { categoría }\end{array}$ \\
\hline Transformación & $\begin{array}{l}\text { "(...) Antes se dice } \\
\text { que sólo habían } \\
\text { microorganismos y } \\
\text { a medida que fue } \\
\text { pasando el tiempo, } \\
\text { fueron } \\
\text { transformándose, a } \\
\text { lo que hoy en día } \\
\text { conocemos } \\
\text { ecosistemas" } \\
\text { (E002) }\end{array}$ & $\begin{array}{l}\text { - Los estudiantes } \\
\text { reconocen el } \\
\text { proceso evolutivo } \\
\text { como una serie de } \\
\text { cambios } \\
\text { progresivos que } \\
\text { se dan en el } \\
\text { transcurso del } \\
\text { tiempo. }\end{array}$ & $\begin{array}{c}12 \\
\text { estudiantes } \\
\text { hacen } \\
\text { referencia } \\
\text { explícita a } \\
\text { esta } \\
\text { categoría. }\end{array}$ \\
\hline
\end{tabular}


Edición Extra-Ordinaria. ISSN 2027-1034 P.p 780 - 804

Memorias del VII Encuentro Nacional de Experiencias en la Enseñanza de la Biología y la Educación Ambiental y II Congreso Nacional de Investigación en la Enseñanza de la Biología

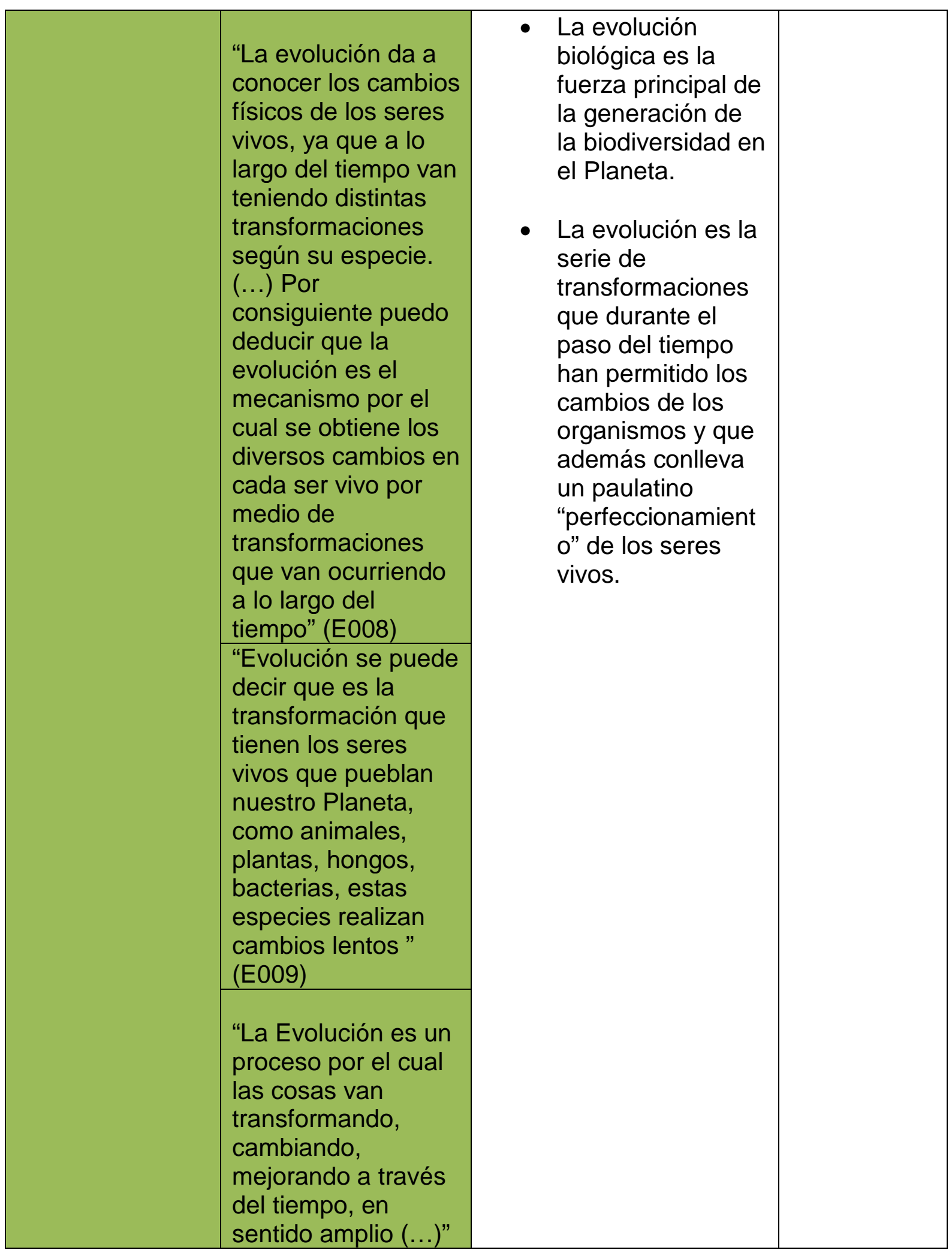


Memorias del VII Encuentro Nacional de Experiencias en la Enseñanza de la Biología y la Educación Ambiental y II Congreso Nacional de Investigación en la Enseñanza de la Biología

\begin{tabular}{|c|c|c|c|}
\hline & (E014) & & \\
\hline & $\begin{array}{l}\text { "En sentido general } \\
\text { evolución es } \\
\text { transformación pero } \\
\text { en sentido biológico } \\
\text { es como cambia los } \\
\text { seres humanos y } \\
\text { por ella estamos } \\
\text { hoy como humanos } \\
\text { en este mundo" } \\
\text { (E019) }\end{array}$ & & \\
\hline $\begin{array}{c}\text { Aparición de } \\
\text { nuevas } \\
\text { especies } \\
\text { (diversidad, } \\
\text { descendencia, } \\
\text { genética) }\end{array}$ & $\begin{array}{l}\text { "Es el conjunto de } \\
\text { cambios y } \\
\text { transformaciones a } \\
\text { través del tiempo } \\
\text { que origina } \\
\text { diversidades de } \\
\text { formas de vida. La } \\
\text { palabra evolución } \\
\text { significa Evo- época } \\
\text { pasada, lución- } \\
\text { Cambio y es } \\
\text { utilizada para } \\
\text { describir los } \\
\text { cambios de toda la } \\
\text { historia" (E018) } \\
\text { "La Evolución es } \\
\text { aquel proceso por el } \\
\text { cual los seres vivos } \\
\text { descienden (...) } \\
\text { según la teoría que } \\
\text { nos plantea Darwin } \\
\text { los seres humanos } \\
\text { descendemos de } \\
\text { los gorilas o } \\
\text { chimpancés de ahí } \\
\text { surge la teoría de la } \\
\text { Evolución" (E020) } \\
\text { "Es una } \\
\text { transformación } \\
\text { progresiva, } \\
\text { transformarse poco }\end{array}$ & $\begin{array}{l}\text { - La Evolución está } \\
\text { relacionada con } \\
\text { las condiciones } \\
\text { genéticas, sin } \\
\text { embargo, no hay } \\
\text { una referencia } \\
\text { explícita de cómo } \\
\text { se relacionan } \\
\text { evolución y } \\
\text { genética. } \\
\text { La evolución } \\
\text { origina nuevas } \\
\text { formas de vida en } \\
\text { el transcurso del } \\
\text { tiempo y las } \\
\text { generaciones. } \\
\text { Los cambios en } \\
\text { los organismos } \\
\text { han permitido dar } \\
\text { origen a la teoría } \\
\text { evolutiva. } \\
\text { Los cambios se } \\
\text { originan por la } \\
\text { capacidad que } \\
\text { tienen los } \\
\text { organismos de }\end{array}$ & $\begin{array}{c}7 \\
\text { estudiantes } \\
\text { en esta } \\
\text { categoría }\end{array}$ \\
\hline
\end{tabular}


Edición Extra-Ordinaria. ISSN 2027-1034 P.p 780 - 804

Memorias del VII Encuentro Nacional de Experiencias en la Enseñanza de la Biología y la Educación Ambiental y II Congreso Nacional de Investigación en la Enseñanza de la Biología

\begin{tabular}{|c|c|c|c|}
\hline & $\begin{array}{l}\text { a poco, a través del } \\
\text { tiempo que han } \\
\text { originado la } \\
\text { diversidad sobre un } \\
\text { antepasado" (E048) } \\
\text { "La evolución es lo } \\
\text { que nosotros } \\
\text { dejamos como una } \\
\text { herencia o algo así } \\
\text { y es lo que hace } \\
\text { que nos } \\
\text { parezcamos a } \\
\text { nuestra familia por } \\
\text { la genética, que lo } \\
\text { vemos en el ADN } \\
\text { (...) A lo largo del } \\
\text { tiempo nosotros } \\
\text { vamos dejando un } \\
\text { parecido en nuestra } \\
\text { familia pero siempre } \\
\text { van surgiendo } \\
\text { grandes cambios" } \\
\text { (E035) }\end{array}$ & $\begin{array}{l}\text { dejar } \\
\text { descendencia, en } \\
\text { el transcurso del } \\
\text { tiempo se pueden } \\
\text { ver grandes } \\
\text { cambios. }\end{array}$ & \\
\hline Mejoramiento & $\begin{array}{l}\text { "Es el desarrollo } \\
\text { secuencial de un } \\
\text { suceso mediante el } \\
\text { cual se presentan } \\
\text { cambios en pro de } \\
\text { una situación" } \\
\text { (E017) } \\
\text { "Puede entenderse } \\
\text { como un proceso } \\
\text { que deben } \\
\text { atravesar algunos y } \\
\text { que consiste en el } \\
\text { abandono de una } \\
\text { etapa a otra, es } \\
\text { también una etapa } \\
\text { de mejora que cada } \\
\text { vez más se vuelve } \\
\text { casi perfecto" } \\
\text { (E021) } \\
\text { "La evolución es el }\end{array}$ & $\begin{array}{l}\text { - Los estudiantes } \\
\text { manifiestan que la } \\
\text { evolución es la } \\
\text { fuerza que } \\
\text { conduce al } \\
\text { mejoramiento } \\
\text { continuo de los } \\
\text { organismos, es la } \\
\text { ruta que conduce } \\
\text { hacia la perfección } \\
\text { - El mejoramiento } \\
\text { en los organismos } \\
\text { conlleva un } \\
\text { mejoramiento en } \\
\text { la calidad de vida } \\
\text { y por tanto en la } \\
\text { cantidad de }\end{array}$ & $\begin{array}{c}10 \\
\text { estudiantes } \\
\text { en esta } \\
\text { categoría }\end{array}$ \\
\hline
\end{tabular}


Bio-grafia Escritos sobre la Biología y su Enseñanza.

Edición Extra-Ordinaria. ISSN 2027-1034 P.p 780 - 804

Memorias del VII Encuentro Nacional de Experiencias en la Enseñanza de la Biología y la Educación Ambiental y II Congreso Nacional de Investigación en la Enseñanza de la Biología

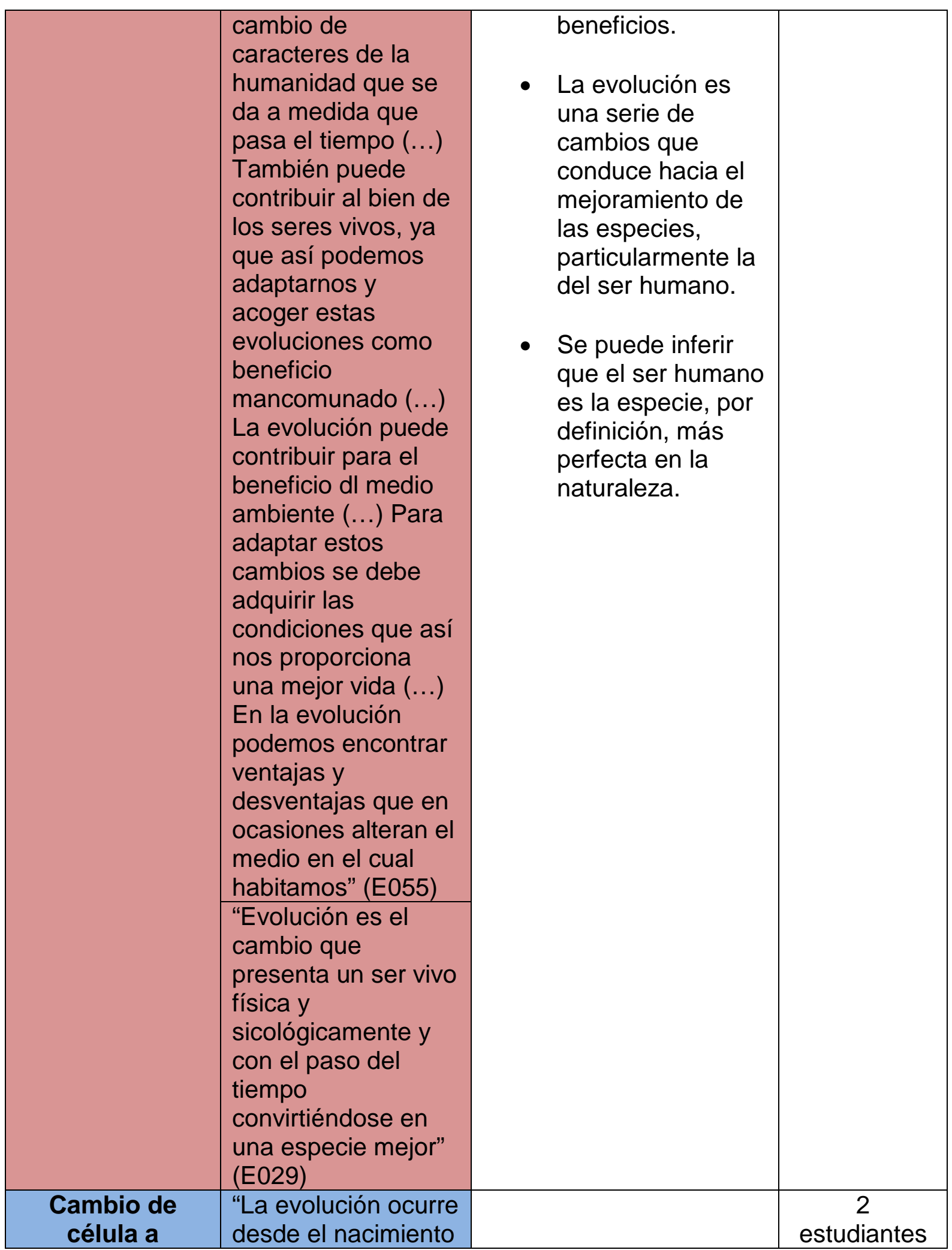


Memorias del VII Encuentro Nacional de Experiencias en la Enseñanza de la Biología y la Educación Ambiental y II Congreso Nacional de Investigación en la Enseñanza de la Biología

\begin{tabular}{|c|c|c|c|}
\hline $\begin{array}{l}\text { organismos } \\
\text { complejos }\end{array}$ & $\begin{array}{l}\text { de la primera célula } \\
\text { en el planeta Tierra } \\
\text { debido a que desde } \\
\text { ese momento le han } \\
\text { ocurrido cambios a } \\
\text { todos los que } \\
\text { habitan la Tierra, } \\
\text { cambios físicos que } \\
\text { funcionan para } \\
\text { adaptarse el } \\
\text { individuo al } \\
\text { ambiente, por eso } \\
\text { los seres } \\
\text { evolucionamos" } \\
\text { (E037) } \\
\text { "Todas las especies } \\
\text { evolucionaron de } \\
\text { una sola célula de } \\
\text { la cual salieron } \\
\text { varias especies, y } \\
\text { estas evolucionaron } \\
\text { para convertirse en } \\
\text { lo que hoy } \\
\text { conocemos" (E036) }\end{array}$ & $\begin{array}{l}\text { - La complejidad de } \\
\text { los organismos se } \\
\text { originó a partir de } \\
\text { una célula, que en } \\
\text { el tiempo fue } \\
\text { cambiando, } \\
\text { complejizándose } \\
\text { hasta alcanzar las } \\
\text { formas diversas } \\
\text { que se conocen } \\
\text { actualmente }\end{array}$ & $\begin{array}{c}\text { en esta } \\
\text { categoría }\end{array}$ \\
\hline $\begin{array}{c}\text { Cambios } \\
\text { durante la vida } \\
\text { de un } \\
\text { organismo } \\
\text { (Desarrollo) }\end{array}$ & $\begin{array}{l}\text { "Son cambios } \\
\text { graduales que a } \\
\text { través del tiempo se } \\
\text { han id } \\
\text { transformando, los } \\
\text { organismos } \\
\text { pasando de un } \\
\text { estado a otro a } \\
\text { medida del tiempo } \\
\text { se van } \\
\text { desarrollando cada } \\
\text { vez más y más Ya } \\
\text { que diferentes } \\
\text { especies de } \\
\text { organismos han ido } \\
\text { evolucionando al } \\
\text { transcurso del } \\
\text { tiempo." (E031) }\end{array}$ & $\begin{array}{l}\text { - Los estudiantes } \\
\text { explican que la } \\
\text { evolución es el } \\
\text { conjunto de } \\
\text { cambios que } \\
\text { tienen los } \\
\text { individuos a lo } \\
\text { largo d su vida, } \\
\text { estos cambios no } \\
\text { sólo son a nivel } \\
\text { físico, sino que } \\
\text { también conllevan } \\
\text { cambios } \\
\text { emocionales y } \\
\text { sicológicos. } \\
\text { Resulta evidente } \\
\text { que la concepción }\end{array}$ & $\begin{array}{c}5 \\
\text { estudiantes } \\
\text { en esta } \\
\text { categoría }\end{array}$ \\
\hline
\end{tabular}


Memorias del VII Encuentro Nacional de Experiencias en la Enseñanza de la Biología y la Educación Ambiental y II Congreso Nacional de Investigación en la Enseñanza de la Biología

\begin{tabular}{|c|c|c|c|}
\hline & $\begin{array}{l}\text { "La evolución son } \\
\text { una serie de } \\
\text { cambios que nacen } \\
\text { a raíz de una cosa, } \\
\text { que con el paso del } \\
\text { tiempo va sufriendo } \\
\text { unos cambios ya } \\
\text { sea por razones de } \\
\text { genética (...) El otro } \\
\text { cambio se debe a la } \\
\text { genética que con } \\
\text { los cambios que se } \\
\text { producen el ADN y } \\
\text { en el cuerpo pasa a } \\
\text { formar parte de otra } \\
\text { especie, como lo es } \\
\text { el renacuajo que } \\
\text { pasa por una serie } \\
\text { de cambios hasta } \\
\text { que termina } \\
\text { convertido en sapo " } \\
\text { (E038) } \\
\text { "La evolución } \\
\text { significa desarrollo y } \\
\text { es como la } \\
\text { transformación de } \\
\text { algo, la evolución el } \\
\text { principio biológico } \\
\text { habla de los seres } \\
\text { vivos, animales y } \\
\text { vegetales etc. (...) } \\
\text { También es como el } \\
\text { desarrollo de las } \\
\text { cosas o de los } \\
\text { organismos por } \\
\text { medio del cual } \\
\text { pasan de un estado } \\
\text { a otro" (E052) }\end{array}$ & $\begin{array}{l}\text { de algunos } \\
\text { estudiantes sobre } \\
\text { la evolución se } \\
\text { comprende como } \\
\text { el cambio durante } \\
\text { el cambio de vida, } \\
\text { es decir, se } \\
\text { relaciona el } \\
\text { proceso evolutivo } \\
\text { con los procesos } \\
\text { de metamorfosis. } \\
\text { En este sentido la } \\
\text { evolución } \\
\text { represente la } \\
\text { ontogenia de los } \\
\text { organismos. }\end{array}$ & \\
\hline $\begin{array}{c}\text { Cambios } \\
\text { físicos, } \\
\text { mentales. En el } \\
\text { tiempo }\end{array}$ & $\begin{array}{l}\text { "Evolución es el } \\
\text { cambio que } \\
\text { presenta un ser } \\
\text { vivo, física y }\end{array}$ & $\begin{array}{l}\text { - El cambio en los } \\
\text { organismos está } \\
\text { sujeto a las } \\
\text { condiciones en las }\end{array}$ & $\begin{array}{c}23 \\
\text { estudiantes } \\
\text { en esta } \\
\text { categoría }\end{array}$ \\
\hline
\end{tabular}


Bio-grafia Escritos sobre la Biología y su Enseñanza.

Edición Extra-Ordinaria. ISSN 2027-1034 P.p 780 - 804

Memorias del VII Encuentro Nacional de Experiencias en la Enseñanza de la Biología y la Educación Ambiental y II Congreso Nacional de Investigación en la Enseñanza de la Biología

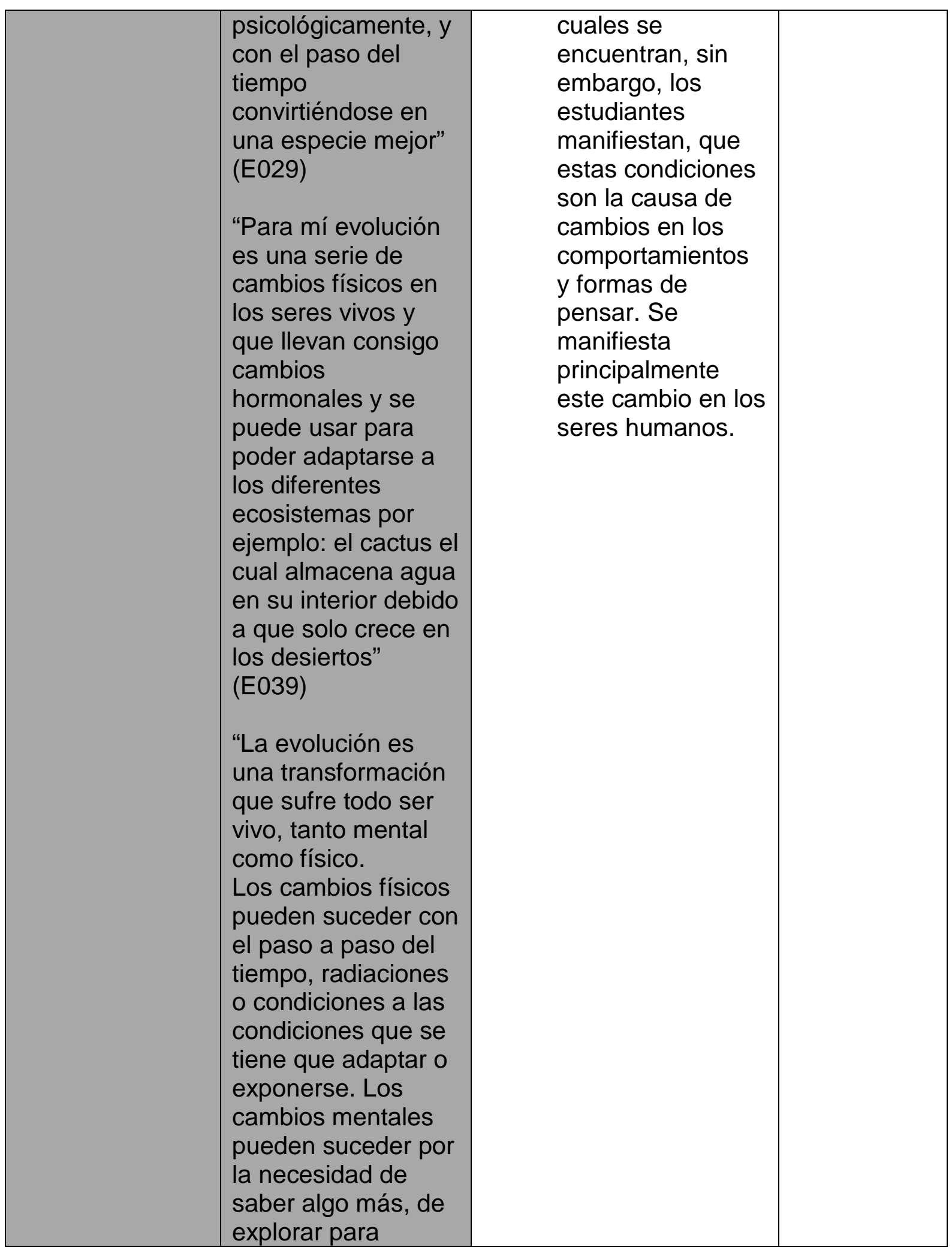


Memorias del VII Encuentro Nacional de Experiencias en la Enseñanza de la Biología y la Educación Ambiental y II Congreso Nacional de Investigación en la Enseñanza de la Biología

\begin{tabular}{|c|c|c|c|}
\hline & $\begin{array}{l}\text { conocer qué es lo } \\
\text { que pasa, para que } \\
\text { sirve y otros tipos } \\
\text { de experimentación. } \\
\text { (...) Se puede } \\
\text { deducir que la } \\
\text { evolución son } \\
\text { cambios que sufre } \\
\text { un ser vivo sean } \\
\text { físicos y mentales } \\
\text { con el paso del } \\
\text { tiempo, o diferentes } \\
\text { adaptaciones que } \\
\text { tienen que vivir" } \\
\text { (E024) }\end{array}$ & & \\
\hline Adaptación & $\begin{array}{l}\text { "La evolución es el } \\
\text { cambio de } \\
\text { adaptación en un } \\
\text { ser u objeto a las } \\
\text { circunstancias que } \\
\text { lo rodean, es la } \\
\text { adaptación de un } \\
\text { ser vivo a un medio } \\
\text { ambiente que está } \\
\text { en constante } \\
\text { cambio" (E006) } \\
\text { "Los seres vivos } \\
\text { comparten } \\
\text { diferentes formas de } \\
\text { vida que dependen } \\
\text { del clima, las alturas } \\
\text { o las profundidades, } \\
\text { las estaciones, el } \\
\text { suelo y los } \\
\text { accidenten del } \\
\text { terreno. Estas } \\
\text { condiciones son las } \\
\text { que hacen variar las } \\
\text { oportunidades y } \\
\text { dificultades para } \\
\text { vivir y adaptarse a } \\
\text { diferentes sitios" }\end{array}$ & $\begin{array}{l}\text { - Según manifiestan } \\
\text { los estudiantes, la } \\
\text { adaptación como } \\
\text { resultado de la } \\
\text { evolución } \\
\text { responde a las } \\
\text { necesidades de } \\
\text { los organismos } \\
\text { por habitar y } \\
\text { desarrollar sus } \\
\text { funciones } \\
\text { estructurales en } \\
\text { un ambiente } \\
\text { determinado. } \\
\text { La adaptación es } \\
\text { resultado del } \\
\text { proceso evolutivo, } \\
\text { sin embargo, los } \\
\text { estudiantes, no } \\
\text { hacen referencia a } \\
\text { los eventos y } \\
\text { procesos que } \\
\text { conducen a los } \\
\text { cambios } \\
\text { evolutivos, sino } \\
\text { que éstos }\end{array}$ & $\begin{array}{c}10 \\
\text { estudiantes } \\
\text { en esta } \\
\text { categoría }\end{array}$ \\
\hline
\end{tabular}


Memorias del VII Encuentro Nacional de Experiencias en la Enseñanza de la Biología y la Educación Ambiental y II Congreso Nacional de Investigación en la Enseñanza de la Biología

\begin{tabular}{|c|c|c|c|}
\hline & $\begin{array}{l}\text { (E047) } \\
\text { "La evolución se } \\
\text { presenta en las } \\
\text { especies, es un } \\
\text { cambio que hacen } \\
\text { para adaptarse al } \\
\text { medio ambiente, } \\
\text { como el climático. } \\
\text { Este cambio } \\
\text { "Evolución" se } \\
\text { desarrolla porque } \\
\text { cuando su } \\
\text { organismo su forma } \\
\text { física no tiene la } \\
\text { capacidad para } \\
\text { sobrevivir desarrolla } \\
\text { nuevas partes del } \\
\text { cuerpo" (E048) }\end{array}$ & $\begin{array}{l}\text { corresponden a } \\
\text { una necesidad de } \\
\text { los organismos } \\
\text { por evolucionar }\end{array}$ & \\
\hline $\begin{array}{l}\text { Evolución: } \\
\text { Humanos }\end{array}$ & $\begin{array}{l}\text { "La evolución es } \\
\text { como una serie de } \\
\text { transformaciones } \\
\text { que han venido } \\
\text { cambiando a lo } \\
\text { largo del tiempo, } \\
\text { que va } \\
\text { evolucionando a } \\
\text { cada momento en } \\
\text { los seres humanos, } \\
\text { hace mucho tiempo } \\
\text { no éramos humanos } \\
\text { sino éramos como } \\
\text { una especie de } \\
\text { micos, pero a } \\
\text { medida que ha } \\
\text { transcurrido el } \\
\text { tiempo ha llegado a } \\
\text { la evolución de los } \\
\text { humanos" (E010) } \\
\text { "En sentido general } \\
\text { evolución es } \\
\text { transformación, }\end{array}$ & $\begin{array}{l}\text { - Los estudiantes } \\
\text { hacen explícito } \\
\text { que la evolución } \\
\text { es propia, y casi } \\
\text { que exclusiva, del } \\
\text { ser humano. } \\
\\
\text { - A través de la } \\
\text { evolución el } \\
\text { hombre ha } \\
\text { desarrollado un } \\
\text { conjunto de } \\
\text { tecnologías que le } \\
\text { han permitido } \\
\text { mejorar su calidad } \\
\text { de vida, así como } \\
\text { también ha traído } \\
\text { perjuicios para sí } \\
\text { mismo. }\end{array}$ & $\begin{array}{c}16 \\
\text { estudiantes } \\
\text { en esta } \\
\text { categoría }\end{array}$ \\
\hline
\end{tabular}


Bio-grafia Escritos sobre la Biología y su Enseñanza.

Edición Extra-Ordinaria. ISSN 2027-1034 P.p 780 - 804

Memorias del VII Encuentro Nacional de Experiencias en la Enseñanza de la Biología y la Educación Ambiental y II Congreso Nacional de Investigación en la Enseñanza de La Biología

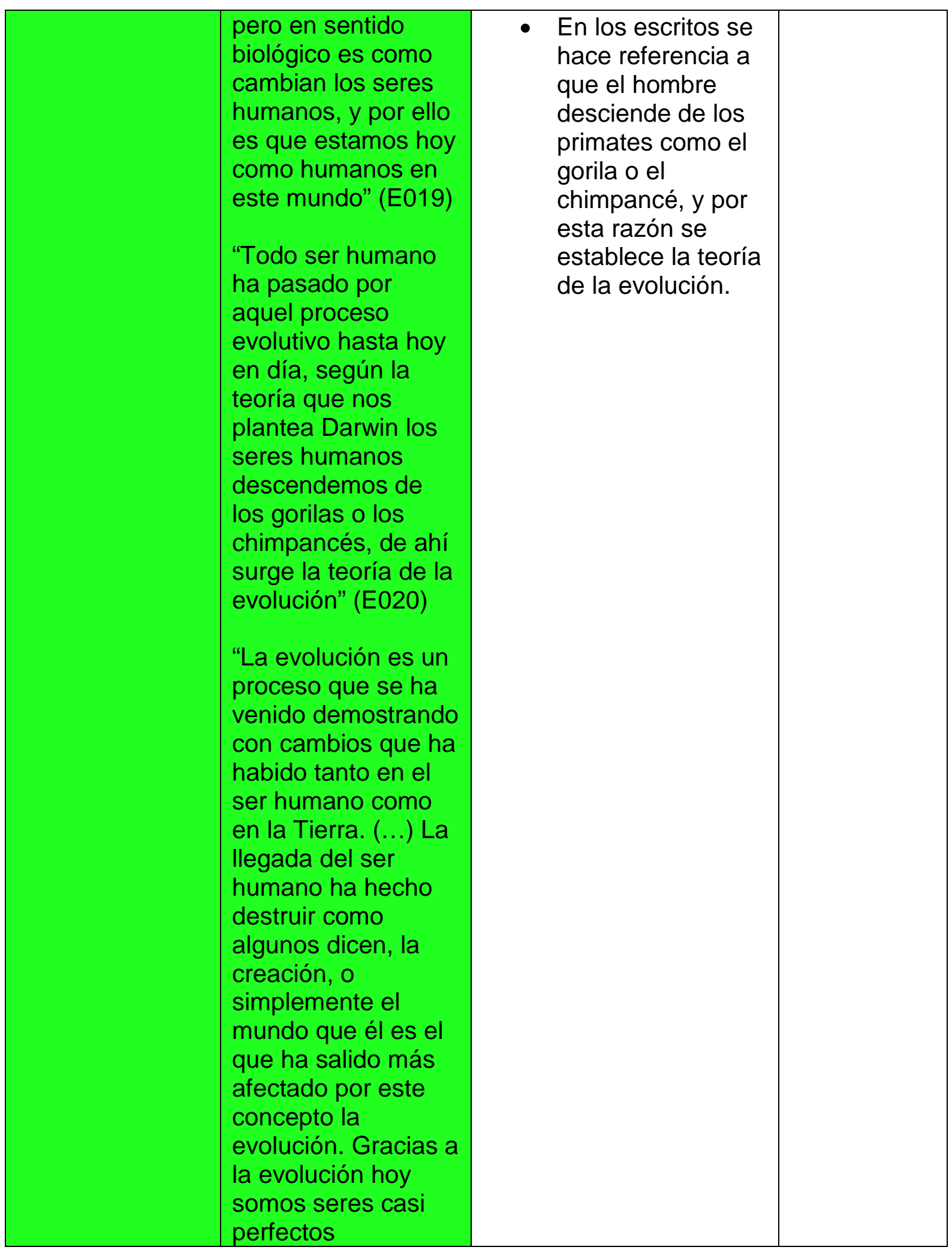


Memorias del VII Encuentro Nacional de Experiencias en la Enseñanza de la Biología y la Educación Ambiental y II Congreso Nacional de Investigación en la Enseñanza de la Biología

\begin{tabular}{|c|c|c|c|}
\hline & $\begin{array}{l}\text { físicamente aunque } \\
\text { esto también ha } \\
\text { traído } \\
\text { enfermedades que } \\
\text { son propagadas por } \\
\text { algunos animales o } \\
\text { los mismos seres } \\
\text { vivos" (E062) }\end{array}$ & & \\
\hline $\begin{array}{c}\text { Avance } \\
\text { tecnológico }\end{array}$ & $\begin{array}{l}\text { "La evolución ha } \\
\text { sido y será muy } \\
\text { importante en } \\
\text { nuestra vida diaria, } \\
\text { porque cada día las } \\
\text { persones piensan } \\
\text { diferentes cosas, } \\
\text { como inventos para } \\
\text { ayudar a los demás } \\
\text { y facilitar la vida } \\
\text { cotidiana sin utilizar } \\
\text { métodos antiguos. } \\
\text { La evolución del } \\
\text { hombre es igual } \\
\text { porque han crecido } \\
\text { y desarrollado sus } \\
\text { cuerpos; existen } \\
\text { personas que no } \\
\text { necesitan la ayuda } \\
\text { de sus padres para } \\
\text { ser creados sino } \\
\text { son creados por } \\
\text { nuevos métodos, } \\
\text { donde el humano } \\
\text { manipula la } \\
\text { tecnología de la } \\
\text { ciencia para dar } \\
\text { vida a un nuevo ser" } \\
\text { (E034) } \\
\text { "En otras palabras } \\
\text { evolucionar es } \\
\text { también innovar, ser } \\
\text { mejores. El hombre }\end{array}$ & $\begin{array}{l}\text { - La evolución es el } \\
\text { resultado del } \\
\text { ingenio del } \\
\text { hombre, por tanto } \\
\text { el desarrollo de } \\
\text { nuevas } \\
\text { tecnologías ha } \\
\text { permitido el } \\
\text { mejoramiento de } \\
\text { la calidad de vida } \\
\text { de la especie. } \\
\text { La evolución es un } \\
\text { proceso de } \\
\text { innovación que } \\
\text { permite al hombre } \\
\text { desarrollar } \\
\text { inventos cada vez } \\
\text { mejores con } \\
\text { respecto a las } \\
\text { funciones que } \\
\text { desempeñan los } \\
\text { dispositivos; se } \\
\text { hace referencia a } \\
\text { la miniaturización } \\
\text { de los } \\
\text { procesadores y el } \\
\text { cambio de los } \\
\text { teléfonos } \\
\text { celulares, como el } \\
\text { paso de los } \\
\text { primeros móviles } \\
\text { hasta los }\end{array}$ & $\begin{array}{c}15 \\
\text { estudiantes } \\
\text { en esta } \\
\text { categoría }\end{array}$ \\
\hline
\end{tabular}


Bio-grafia Escritos sobre la Biologia y su Enseñanza.

Edición Extra-Ordinaria. ISSN 2027-1034 P.p 780 - 804

Memorias del VII Encuentro Nacional de Experiencias en la Enseñanza de la Biología y la Educación Ambiental y II Congreso Nacional de Investigación en la Enseñanza de la Biología

\begin{tabular}{|c|c|c|c|}
\hline & $\begin{array}{l}\text { que fue uno de los } \\
\text { evolucionados } \\
\text { también hizo } \\
\text { cambiar el mundo } \\
\text { con sus ideas, no } \\
\text { siendo consecuente } \\
\text { que todas aquellas } \\
\text { transformaciones no } \\
\text { son favorables para } \\
\text { el mundo y por } \\
\text { tanto para sí mismo } \\
\text { " (E040) } \\
\text { "Es un conjunto de } \\
\text { transformaciones } \\
\text { que a medida que } \\
\text { pasa el tiempo van } \\
\text { avanzando y que } \\
\text { como la tecnología } \\
\text { avanza la evolución } \\
\text { de la misma manera } \\
\text { evoluciona. Muchos } \\
\text { casos han pasado a } \\
\text { través de la } \\
\text { evolución, ¿Cómo } \\
\text { cuáles?: Animales } \\
\text { humanidad, } \\
\text { vestuario, } \\
\text { tecnología, } \\
\text { accesorios, etc. Han } \\
\text { progresado a } \\
\text { medida que se } \\
\text { descubren nuevas } \\
\text { cosas, nuevos } \\
\text { descubrimientos, ha } \\
\text { cambiado hasta la } \\
\text { forma de pensar de } \\
\text { la gente (E050)" }\end{array}$ & $\begin{array}{l}\text { denominados } \\
\text { Smart-Phones }\end{array}$ & \\
\hline $\begin{array}{l}\text { Paso por } \\
\text { etapas }\end{array}$ & $\begin{array}{l}\text { "Puede entenderse } \\
\text { como un proceso } \\
\text { que deben } \\
\text { atravesar algunos y } \\
\text { que consiste en el }\end{array}$ & $\begin{array}{l}\text { - Los estudiantes } \\
\text { conservan la } \\
\text { concepción de que } \\
\text { la evolución }\end{array}$ & $\begin{array}{c}12 \\
\text { estudiantes } \\
\text { en esta } \\
\text { categoría }\end{array}$ \\
\hline
\end{tabular}


Edición Extra-Ordinaria. ISSN 2027-1034 P.p 780 - 804

Memorias del VII Encuentro Nacional de Experiencias en la Enseñanza de la Biología y la Educación Ambiental y II Congreso Nacional de Investigación en la Enseñanza de La Biología

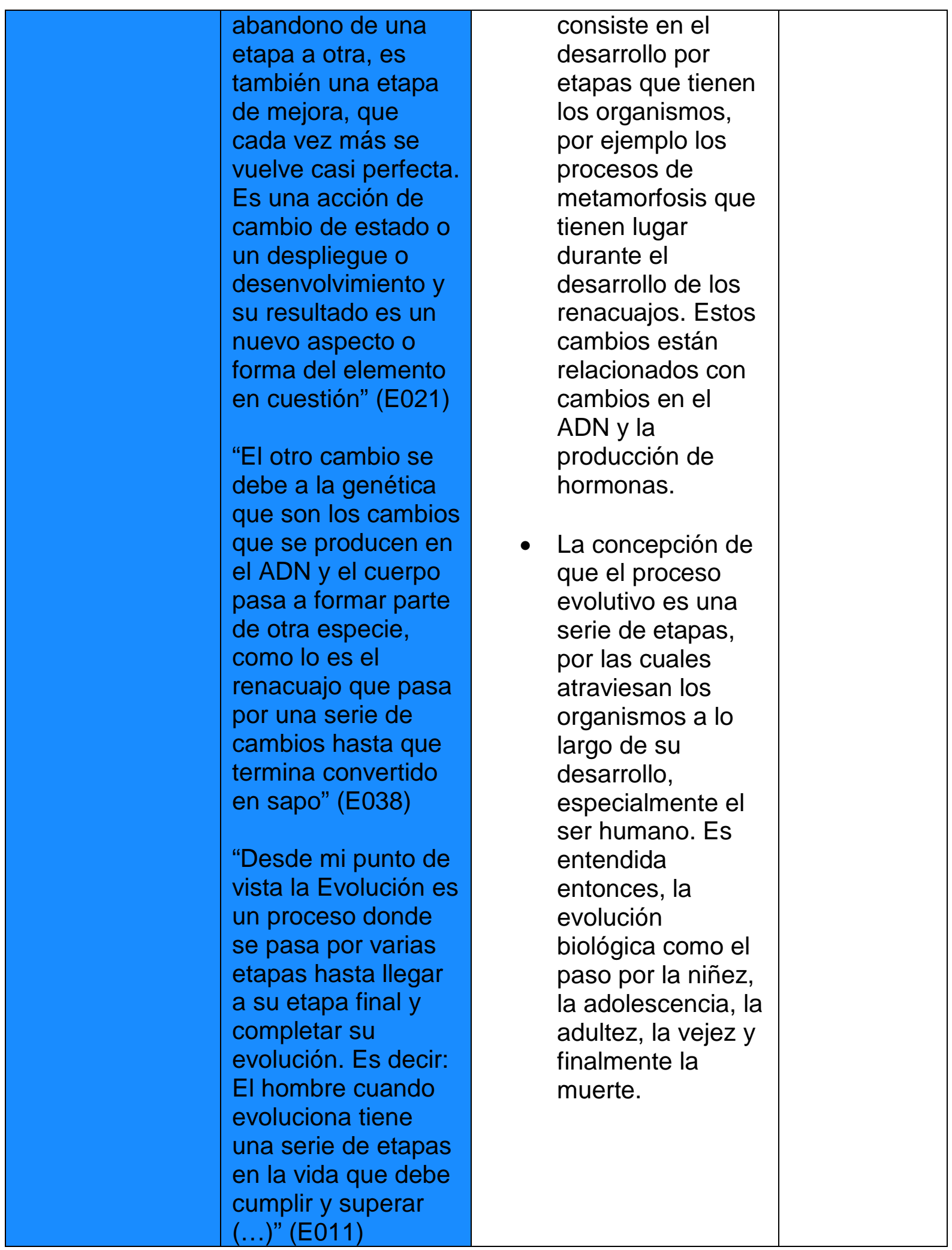


Bio-grafía Escritos sobre la Biología y su Enseñanza.

Edición Extra-Ordinaria. ISSN 2027-1034 P. p 780 - 804

Memorias del VII Encuentro Nacional de Experiencias en la Enseñanza de la Biología y la Educación Ambiental y II Congreso Nacional de Investigación en la Enseñanza de la Biología

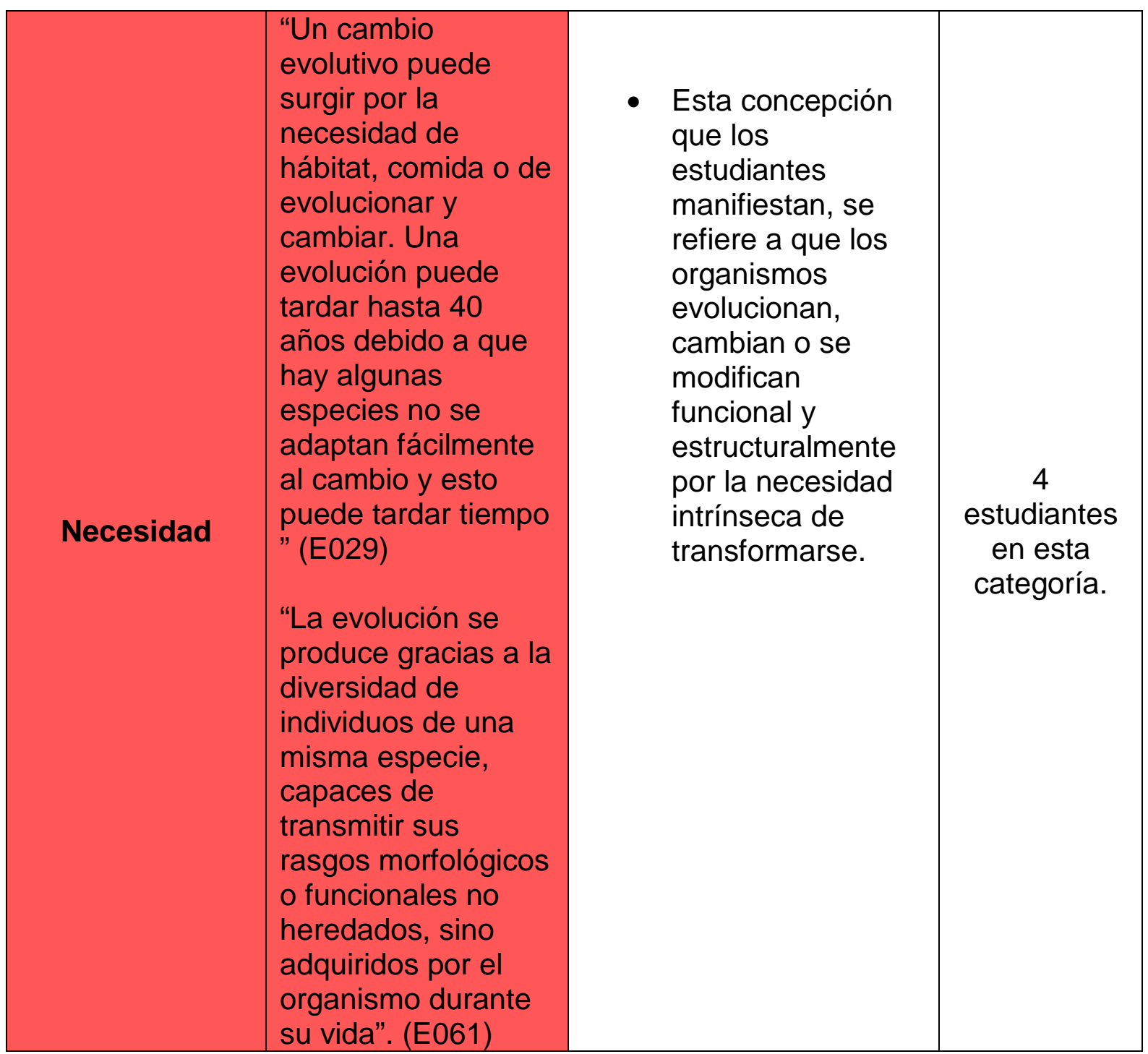

